\title{
FEASIBILITY STUDY OF VIBRATION ABSORBERS WITH SIMPLIFIED SHIP PROPULSION MODELS
}

\author{
M. Loccufier F. Petit D. Aeyels
}

SYSTeMS Research Group

Technologiepark Zwijnaarde 9149052 Zwijnaarde

\begin{abstract}
Torsional vibrations on the propulsion drive train of a ship due to harmonic loads are thoroughly analyzed by classification societies. During operation however the drive train is subjected to transient loads and the masselastic system interacts with the speed controller. Here we derive a smart reduced model of the ship propulsion system capable of revealing the low frequent dynamics which dominate the transient response. A possible approach to counter the damage caused by transient loads, is the attachment of a vibration absorber. The simplified model allows us to check the feasibility of this approach.Copyright (c) 2007 IFAC
\end{abstract}

Keywords: Model reduction, ship control, vibration dampers, transient responses, torsional vibration

\section{INTRODUCTION}

The drive train of a ship is a complex assembly of a diesel engine, a generator, a propeller, transmission gears, clutches, steel shafts, flexible couplings...A torsional vibration is an oscillatory twisting of these elements superimposed on the steady state rotational motion. The modelling of a torsional mass-elastic system is a difficult task. The various components must be described in terms of inertias elastically connected with each other. The techniques to do so are well known (Vance, 1988), (Adams, 2001), (Wilson, 1942). The modelling process results in a finite element model:

$$
\mathbf{M}_{\mathrm{m}} \ddot{\Theta}_{\mathrm{m}}+\mathrm{C}_{\mathrm{m}} \dot{\Theta}_{\mathrm{m}}+\mathbf{K}_{\mathrm{m}} \Theta_{\mathrm{m}}=\mathbf{T}_{\mathrm{m}}
$$

This type of model is used in the torsional vibration calculation (TVC) to be approved by the classification society. Based on the expected excitations of the diesel engine, the propeller, the pump, the gears..., calculations are made in the frequency domain to find out if resonance conditions are present (Harris and Piersol, 2001).
When well designed, no excessive vibrations due to harmonic loads should occur. This analysis does not take into account the presence of transient loads nor the effect of the speed controller. Based on the nonuniform inertia and elasticity properties of the ship propulsion system we derive a model reduction technique capable of analyzing the transient response (Section 2). In Section 3 we discuss how this model can be refined. Section 4 explains why the speed controller is not capable of damping the vibration modes excited by suddenly applied loads. In Section 5 we discuss a possible approach to counter transient vibrations: the vibration absorber. Finally an example is given in Section 6 .

\section{MODEL REDUCTION}

The drive train of a large ship is an assembly of shafts rotating at different speeds. The alignment of the shafts is provided by flexible couplings. The stiffness of those flexible couplings is much lower than the stiffness of the steel parts. As a consequence, the lowest eigenfrequencies of the 
complete finite element model will be much lower than the lowest eigenfrequencies of the shafts separately. The transient behaviour of the drive train will be dominated by those low eigenfrequencies. Using the dynamic properties of the flexible couplings, a simple model is derived that gives a good estimate of the low frequent behaviour of the drive train. Let $r-1$ be the number of elastic couplings in the drive train. We derive an undamped model of dimension $r$, i.e. the number of elastic couplings plus one and use this model to estimate the elastic eigenfrequencies $\omega_{2}, \ldots, \omega_{r}$. The lowest eigenfrequency $\left(\omega_{1}=0\right)$ corresponds to the rigid body movement where all inertias rotate at the nominal speed and do not twist against each other. During the reduction technique the following steps are carried out:

(1) All parameters, i.e. inertias $m$ and stiffnesses $k$ are referred to the nominal speed of the shaft that contains the diesel engine. Therefor we calculate the equivalent stiffness $k_{e q}$ and the equivalent inertia $m_{e q}$ of all parameters as follows :

$$
\left(m_{e q}, k_{e q}\right)=\left(\frac{\text { speed shaft }}{\text { speed diesel }}\right)^{2} \times(m, k)
$$

(2) A flexible coupling consists of different segments. We reduce every flexible coupling to an element with two inertias $\mathbf{M}_{\mathbf{p}}(1,1)$ and $\mathbf{M}_{\mathbf{p}}(2,2)$ connected via a massless spring $k_{t_{e}}=\mathbf{K}_{\mathbf{p}}(1,1)$ by using a static reduction technique introduced by Guyan (Guyan, 1965). Fig. 1 illustrates the procedure for a coupling with two segments with torsional stiffnesses $k_{t_{1}}$ and $k_{t_{2}}$ and inertias $m_{C_{1}}$, $m_{C_{2}}$ and $m_{C_{3}}$. First the general principles of Guyan reduction are illustrated, then the technique is applied to the flexible coupling. In static reduction the degrees of freedom

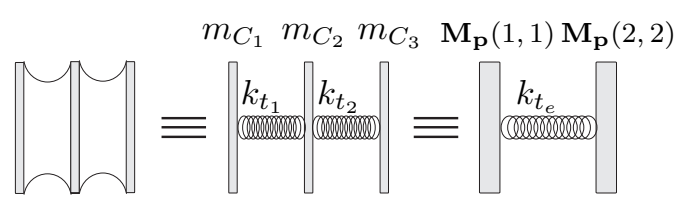

Fig. 1. Reduction of a flexible coupling

of the full model are subdivided into primary and secondary degrees of freedom. The primary degrees of freedom $\theta_{\mathbf{p}}$ are retained while the secondary degrees of freedom $\theta_{\mathbf{s}}$ are eliminated by looking at the static deformation. Consider the equations of motion of an undamped model:

$$
\mathbf{M}_{\mathbf{n}} \ddot{\Theta}_{\mathbf{n}}+\mathbf{K}_{\mathbf{n}} \Theta_{\mathbf{n}}=\mathbf{T}_{\mathbf{n}}
$$

Choosing a set of primary and secondary degrees of freedom yields:

$$
\left[\begin{array}{cc}
\mathbf{M}_{\mathbf{p p}} & 0 \\
0 & \mathbf{M}_{\mathbf{s s}}
\end{array}\right]\left[\begin{array}{l}
\ddot{\Theta}_{\mathbf{p}} \\
\ddot{\Theta}_{\mathbf{s}}
\end{array}\right]+\left[\begin{array}{ll}
\mathbf{K}_{\mathbf{p p}} & \mathbf{K}_{\mathbf{p s}} \\
\mathbf{K}_{\mathbf{s p}} & \mathbf{K}_{\mathbf{s s}}
\end{array}\right]\left[\begin{array}{c}
\boldsymbol{\Theta}_{\mathbf{p}} \\
\boldsymbol{\Theta}_{\mathbf{s}}
\end{array}\right]=\left[\begin{array}{c}
\mathbf{T}_{\mathbf{p p}} \\
\mathbf{0}
\end{array}\right]
$$

Subscripts $p$ and $s$ correspond to the primary and secondary degrees of freedom with $p+$ $s=n$. It is assumed no force is applied to the secondary degrees of freedom. Static reduction implies that the inertia forces of the secondary degrees of freedom are neglected, or:

$$
\mathbf{K}_{\mathrm{sp}} \Theta_{\mathrm{p}}+\mathbf{K}_{\mathrm{ss}} \Theta_{\mathrm{s}}=\mathbf{0} \quad \Rightarrow \Theta_{\mathrm{s}}=\mathbf{S}_{\mathbf{d}} \Theta_{\mathrm{p}}
$$

where $\mathbf{S}_{\mathbf{d}}=-\mathbf{K}_{\mathbf{s s}}{ }^{-1} \mathbf{K}_{\mathbf{s p}}$. The transformation matrix $\mathbf{S}_{\mathbf{f}}$ describes the static transformation between the degrees of freedom of the full model and the primary degrees of freedom:

$$
\left[\begin{array}{l}
\boldsymbol{\Theta}_{\mathbf{p}} \\
\boldsymbol{\Theta}_{\mathbf{s}}
\end{array}\right]=\left[\begin{array}{c}
\mathbf{I} \\
-\mathbf{K}_{\mathbf{s s}}{ }^{-1} \mathbf{K}_{\mathbf{s p}}
\end{array}\right]\left[\boldsymbol{\Theta}_{\mathbf{p}}\right]=\mathbf{S}_{\mathbf{f}}\left[\boldsymbol{\Theta}_{\mathbf{p}}\right]
$$

A reduced model can now be obtained with mass and stiffness matrices $\mathbf{M}_{\mathbf{p}}, \mathbf{K}_{\mathbf{p}} \in R^{p \times p}$ :

$$
\mathbf{M}_{\mathbf{p}}=\mathbf{S}_{\mathbf{f}}^{T} \mathbf{M}_{\mathbf{n}} \mathbf{S}_{\mathbf{f}} \quad \mathbf{K}_{\mathbf{p}}=\mathbf{S}_{\mathbf{f}}^{T} \mathbf{K}_{\mathbf{n}} \mathbf{S}_{\mathbf{f}}
$$

where $p<n$ is the number of primary degrees of freedom. In order to apply the Guyan reduction to the flexible coupling we consider it as a subsystem with $n$ elements decoupled from the rest of the drive train (Figure 2). We choose both ends of the coupling as the primary degrees of freedom $(p=2)$. The secondary coordinates $(s=n-2)$ in between are condensed out. According to equation

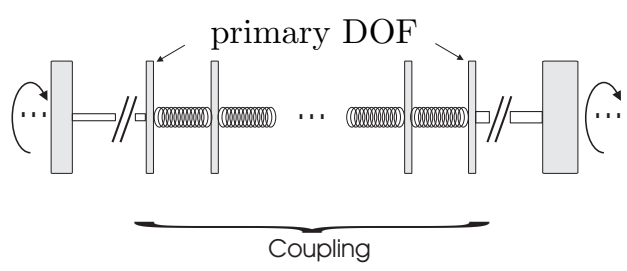

Fig. 2. Reduction of a flexible coupling

(5) we end up with a reduced model for the flexible coupling with mass and stiffness matrices $\mathbf{M}_{\mathbf{p}}$ and $\mathbf{K}_{\mathbf{p}}$ of dimension $2 \times 2$. Notice that the equivalent spring constant $k_{t_{e}}=\mathbf{K}_{\mathbf{p}}(1,1)$ of the reduced coupling is a series connection of all stiffnesses between the first and the last segment. As the off diagonal elements of $\mathbf{M}_{\mathbf{p}}$ have small impact on the eigenfrequencies, they are neglected. Doing so, the reduced mass matrix of the full system remains diagonal.

(3) All inertias between two flexible couplings are joined in one block (Figure 5). This means that the twist of the elastic modes is neglected between any two rotors which are not connected via a flexible coupling.

The result of the reduction process is an undamped model of dimension $r$, where $r$ equals the number of flexible couplings plus one :

$$
\mathbf{M}_{\mathbf{r}} \ddot{\Theta}_{\mathbf{r}}+\mathbf{K}_{\mathbf{r}} \Theta_{\mathbf{r}}=\mathbf{T}_{\mathbf{r}}
$$


where $\mathbf{M}_{\mathbf{r}}, \mathbf{K}_{\mathbf{r}} \in R^{r \times r}$ and $\boldsymbol{\Theta}_{\mathbf{r}}, \mathbf{T}_{\mathbf{r}} \in R^{r} . \mathbf{M}_{\mathbf{r}}=$ $\mathbf{M}_{\mathbf{r}}^{T}$ is a diagonal matrix. Each diagonal element $\mathbf{M}_{\mathbf{r}}(l, l), 1 \leq l \leq r$ corresponds to a block of added inertias. $\mathbf{K}_{\mathbf{r}}=\mathbf{K}_{\mathbf{r}}{ }^{T}$ contains the elastic connections between the blocks and is positive semi-definite. $\boldsymbol{\Theta}_{\mathbf{r}}$ is the rotation angle of the blocks in between the flexible couplings. We solve the eigenvalue problem:

$$
\begin{aligned}
& \operatorname{det}\left(\mathbf{K}_{\mathbf{r}}-\mathbf{M}_{\mathbf{r}} \omega^{2}\right)=0 \Rightarrow \hat{\omega}_{j} ; j=1 \ldots r(7) \\
& \left(\mathbf{K}_{\mathbf{r}}-\mathbf{M}_{\mathbf{r}} \hat{\omega}_{j}^{2}\right) \hat{e}_{j}=0 \Rightarrow \hat{e}_{j} \in R^{r} ; j=1 \ldots r(8)
\end{aligned}
$$

The resulting values $\hat{\omega}_{j}, j=2 \ldots r$ are close estimates of the exact elastic eigenfrequencies $\omega_{j} ; j=2 \ldots r, \hat{\omega}_{1}=\omega_{1}=0$. Note that $(6)$ can be derived by an operator based on the technical drawings of the shaftline.

\section{SENSITIVITY STUDY}

Our main concern is to evaluate the accuracy of the estimates $\hat{\omega}_{j}, j=2 \ldots r$. In our model reduction we assumed every connection besides the flexible couplings to be rigid. We search for the most critical element in the drive train, i.e. the element that violates the most this assumption. The drive train of a ship propulsion system has nonuniform inertia- and stiffness properties. The diesel engine provides the power to rotate all shafts. Every shaft is at one end directly or indirectly connected with the diesel engine. The other end of the shaft, in what follows called the free end, normally bears an important rotor. Important in the sense of the functionality of the rotor (propeller, sand pump, ...), but also important in the modelling process as the mass moment of inertia $m_{r_{e}}$ of such a rotor is often high and the steel parts (with stiffness $k_{r_{e}}$ ) connecting it to neighbouring rotors (clutch, gearbox) can be long, thus reducing $k_{r_{e}}$.

Due to a lower ratio $\frac{k_{r_{e}}}{m_{r_{e}}}$ compared to the other elements in the drive train, these free end rotors are important candidates to extend and improve our model. To understand this we extend the reduced model given in (6) with one degree of freedom $\theta_{\text {re }}$, i.e. one of the free end rotors is decoupled from his block (Fig. 3). This means the dimension of mass- and stiffness matrices $\mathbf{M}_{\mathbf{r}}$ and $\mathbf{K}_{\mathbf{r}}$ is changed to $R^{(r+1) \times(r+1)}$. The new vibration

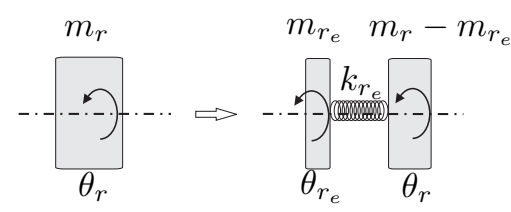

Fig. 3. Model extension

shape near $\theta_{r e}$ is given by an extra equation in (8)

$$
-k_{r e} \theta_{r}+\left(k_{r e}-m_{r e} \omega^{2}\right) \theta_{r e}=0
$$

In the reduced model we assumed a rigid connection between the free end rotor and the rest of the drive train. In other words:

$$
\frac{\theta_{r e}}{\theta_{r}}=\frac{k_{r e}}{\left(k_{r e}-m_{r e} \omega^{2}\right)}=1
$$

This assumption will certainly be justified if the ratio

$$
\frac{\omega^{2} m_{r_{e}}}{k_{r_{e}}} \ll 1
$$

for all estimated eigenvalues, i.e. for all values $\hat{\omega}_{j}, j=1 \ldots r$. We use the ratio given in (11) as a first indication for the accuracy of our model. We calculate this ratio for all free end rotors of the complete finite element model. A large ratio indicates that we can improve our model by extending it with the free end rotor. Doing so the equations of motion read :

$$
\mathbf{M}_{\mathbf{r}+\mathbf{1}} \ddot{\Theta}_{\mathbf{r}+\mathbf{1}}+\mathbf{K}_{\mathbf{r}+\mathbf{1}} \Theta_{\mathbf{r}+\mathbf{1}}=\mathbf{T}_{\mathbf{r}+\mathbf{1}}
$$

$\mathbf{M}_{\mathbf{r}+\mathbf{1}}, \mathbf{K}_{\mathbf{r}+\mathbf{1}} \in R^{(r+1) \times(r+1)}$ and $\boldsymbol{\Theta}_{\mathbf{r}+\mathbf{1}}, \mathbf{T}_{\mathbf{r}+\mathbf{1}} \in$ $R^{r+1}$

\section{GOVERNOR INTERACTION}

Transient loads arise for example at engine startup, by random cylinder misfiring and while engaging and disengaging the propeller and the pump. Using modal analysis the response to an impulse with intensity $T_{m_{I}}$ can be decoupled into terms of the form:

$$
\mathbf{e}_{\mathbf{k}}(c) \frac{1}{\omega_{k}} \sin \left(\omega_{k} t\right) \mathbf{e}_{\mathbf{k}}^{T}(b) T_{m_{I}}
$$

Each term corresponds to an eigenfrequency $\omega_{k}$, $\mathbf{e}_{\mathbf{k}}$ is the corresponding eigenvector. $\mathbf{e}_{\mathbf{k}}(i)$ is component $i$ of eigenvector $\mathbf{e}_{\mathbf{k}} \cdot b$ stands for the location of excitation (input), $c$ for the output location. Notice that (13) is proportional with $\frac{1}{\omega_{k}}$. Therefore the transient behaviour will be dominated by the lowest eigenfrequencies. This means we can analyze the qualitative transient response of the ship propulsion system with the reduced model (6).

This analysis is not complete if we do not take into account the interaction of the speed controller with the mass-elastic system. We assume collocated control. This means that the speed pick-up (sensor) is located at the diesel engine (actuator). We restrict ourself to proportional control (with gain $K_{\text {prop }}$ ) of the speed to illustrate the basic ideas. The simplified scheme of the speed control loop is shown on Fig.4. We analyze the effect of the controller with the root locus technique, i.e. we study the shift of the closed loop poles if the proportional gain of the controller is varied from 0 till $\infty$. Fig. 5 shows the several branches corresponding to the different eigenfrequencies of the reduced model (6). We notice one branch on the real axis. This corresponds to the first order 


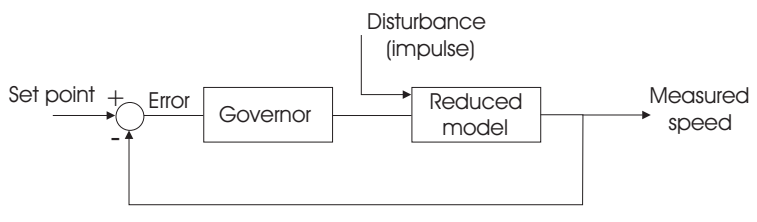

Fig. 4. Control loop

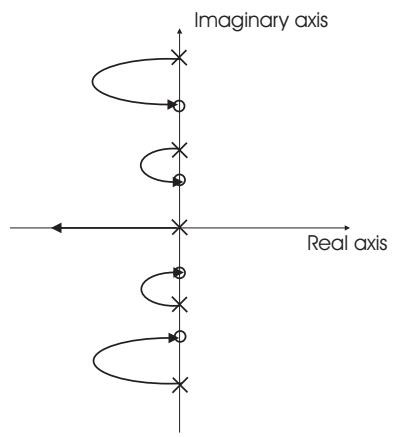

Fig. 5. Root locus with proportional controller

behaviour of the nominal mode introduced by the controller. This mode will dominate the step response and will be used to tune the controller. The other loops are small loops entering the left half plane as $K_{\text {prop }}$ increases and returning to the imaginary axis for $K_{\text {prop }} \rightarrow \infty$. It seems the controller is not capable of damping the elastic modes excited by suddenly applied loads. Introducing I-action and D-action in the controller will change the branch on the real axis of the root locus, but will not change the damping capacities of the controller w.r.t. the elastic modes (Petit and Loccufier, 2006).

\section{VIBRATION ABSORBER}

We study the possibility of damping the elastic modes by attaching an extra passive system to the main propulsion drive system. The simplest passive system is a vibration absorber. Vibration absorbers are common use in a wide range of engineering applications (Koronev and Reznikov, 1993). Although it is most effective for reducing vibration amplitudes arising from periodic excitations, we will study the effect the absorber has on the vibration amplitudes caused by transient loads. First the basic principles of a vibration absorber are explained in steady state conditions. Then we discuss how to design the absorber for transient conditions.

\subsection{Periodic excitations}

Consider a single degree of freedom system (SDOF) excited by a harmonic load with frequency $\omega_{a}$. To reduce vibrations of the main structure we attach a passive element, i.e. the vibration absorber. Basically the absorber consists of an inertia $\left(m_{h}\right)$ connected at a certain location to the structure with springs $\left(k_{h}\right)$ and dampers $\left(c_{h}\right)$ (Fig. 6). The founders of this technique are Frahm (Frahm, 1911) and Den Hartog (Den Hartog, 1956). Although the absorber can reduce vibrations at any excitation frequency, it is most efficient in resonance conditions. This means the excitation frequency $\omega_{a}$ equals one of the eigenfrequencies $\omega_{i}$ of the propulsion system. A vibration absorber tuned to this eigenfrequency $\omega_{i}$, i.e. $\omega_{h}=\sqrt{\frac{k_{h}}{m_{h}}}=\omega_{i}$ greatly reduces the vibration amplitude over a wide frequency band. Fig. 7 shows the vibration amplitude for different values of the damping factor $\zeta$. One can notice two points ( $\mathrm{A}$ and $\mathrm{B}$ ) which are independent of the damping factor $\zeta$. Balancing the vibration amplitudes of these invariant points leads to the well known formulas:

$$
\alpha_{o p t}=\frac{1}{1+\mu} \quad ; \quad \zeta_{\text {opt }}=\sqrt{\frac{\mu}{2(1+\mu)}}
$$

with $\alpha=\frac{\omega_{h}}{\omega_{a}}$ and $\mu=\frac{m_{h}}{m}$. Equation (14) gives the optimum absorber parameters for an undamped single degree of freedom (SDOF) system excited by a harmonic force. The absorber is tuned to the eigenfrequency $\omega_{i}$ of the main system. When the main system is a multi degree of freedom (MDOF) system such as the drive train of a ship, two important differences affect the optimum absorber design. First of all, the influence of neighbouring vibration modes becomes important. Second, the spatial location of the absorber is an extra parameter in the design. A great deal of research has been done to find the optimum parameters of the absorber for the MDOF system (Rade and Valder, 2000). Recently, the design proposed by Den Hartog for a SDOF system is extended to MDOF systems (Ozer and Royston, 2005). It is shown that invariant points also exist when the main system is an undamped MDOF system. Given a certain location of the main system, the optimum absorber parameters are found by balancing the vibration amplitudes of these invariant points. The question remains where to physically attach the absorber. In a first approximation the eigenvector corresponding with eigenfrequency $\omega_{i}$ is used. The location with the highest activity for that mode is considered as the optimum location. In (Vethecan and Subic, 2002) this topic is discussed more thoroughly.

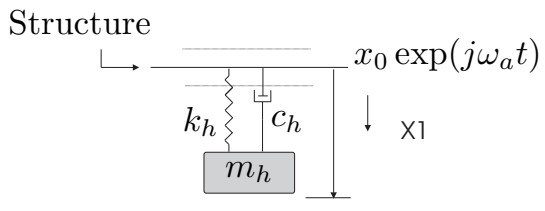

Fig. 6. Translational equivalent of a torsional vibration absorber. 


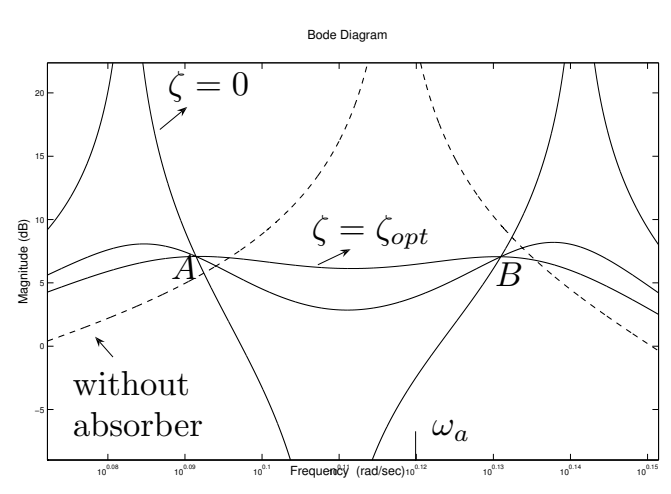

Fig. 7. Attaching a vibration absorber reduces the vibration amplitude near frequency $\omega_{a}$.

\subsection{Transient conditions}

The use of vibration absorbers to cope with periodic excitations is studied for over a hundred years. Recently research is developed in the use of absorbers for transient conditions (Walsh and Lamancusa, 1992). There are important differences in the design of the absorber. When dealing with periodic excitations the vibration absorber is tuned to a single mode. This becomes more complicated when designing the absorber to counter transient effects. This can be seen from equation (13) where different modes contribute to the response of a transient load. This means optimum tuning frequency and location of the absorber are difficult to find. A first approximation would be to tune the absorber to a single mode. As the response is proportional to $\frac{1}{\omega_{k}}$ an obvious choice seems the lowest eigenfrequency. The optimal location remains the same as in the periodic excitation case. This approach is not valid any more when the eigenfrequencies are closely spaced. As a consequence finding the optimum tuning frequency and location for the absorber is quite a challenge. While for the optimal location we could restrict ourself to the mode activity of a single mode in the periodic excitation case, we now have to consider the mode activity of multiple modes. We search for the location $v$ with the highest equivalent mode activity $a_{e q}$ taking into account values of both eigenvector and eigenvalue for the lowest $r$ modes:

$$
a_{e q}=\sum_{i=2}^{r} \frac{\left|\mathbf{e}_{\mathbf{i}}(v)\right|}{\omega_{i}}
$$

We tune the absorber to the eigenfrequency that contributes the most in $a_{e q}$. The use of these design principles are illustrated in the example given in section 6 . Besides the use of a simple vibration absorber other configurations can be investigated. Since multiple modes are excited the use of more complex (multidimensional) passive systems can be studied. Other research fo-

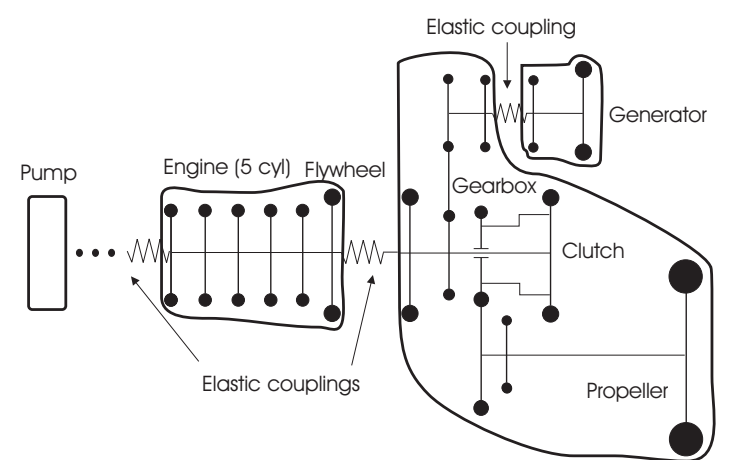

Fig. 8. Model of a ship propulsion system cuses on adaptable vibration absorbers (Walsh and Lamancusa, 1992). This will however not be considered in this article.

\section{EXAMPLE}

Figure 5 shows a finite element model of a ship propulsion system with 20 degrees of freedom (DOF). The drive train consists of 3 flexible couplings combining 4 parts rotating at a different speed. The part on the left of the engine holding the pump is not shown. Every inertia is connected to other inertias through stiffness elements. According to Section 2 a reduced model is obtained with 4 degrees of freedom corresponding with 4 blocks drawn in Figure 8 (part of the pump holds one block). Here the most critical free end rotor as explained in Section 3 is the propeller as can be seen from the ratio $\frac{m_{r_{e}}}{k_{r_{e}}} \omega_{4}^{2}$. Table 1 shows this ratio for every free end rotor. We extend our reduced model with one degree of freedom to obtain a model with 5 degrees of freedom. As can be seen in Table 2 the eigenfrequencies of the reduced model are close estimates of the exact ones. Now the reduced model is used to design a passive system to cope with suddenly applied loads. We consider two transient conditions. One is a step change in the speed of the diesel engine. The other engaging of the dredge pump. We try to reduce the vibration amplitudes near the main elastic coupling, i.e. the coupling between engine and gear box. As discussed in Section 5, we calculate the equivalent mode activity for each possible location of the absorber. We can see that $a_{e q}$ is the highest for the generator (Table 3). As can be seen from the modal matrix given in Table $4 \omega_{3}$ contributes the most to $a_{e q}$. We tune the absorber to this frequency. The absorber configuration is given in Table 5 . The inertia is about $10 \%$ of the generator inertia. Figure 9 shows the reduction the absorber Table 1. $\frac{m_{r_{e}}}{k_{r_{e}}} \omega_{4}^{2}$ for different free end

\begin{tabular}{llll}
\hline Free end rotor & propeller & generator & pump \\
\hline$\frac{m_{r_{e}}}{k_{r_{e}}} \omega_{4}^{2}$ & 0.3843 & 0.06 & 0.001 \\
\hline
\end{tabular}


Table 2. Exact eigenfrequencies of the

FEM model compared with the estimates

\begin{tabular}{llll}
\hline eigenfrequency & exact $\left(\frac{\mathrm{rad}}{\mathrm{s}}\right)$ & estimate $\left(\frac{\mathrm{rad}}{\mathrm{s}}\right)$ & error $(\%)$ \\
\hline$\omega_{1}$ & 0 & 0 & 0 \\
$\omega_{2}$ & 9.6 & 9.47 & 1.37 \\
$\omega_{3}$ & 14.89 & 15.41 & 3.49 \\
$\omega_{4}$ & 20.89 & 21.86 & 4.64 \\
\hline
\end{tabular}

Table 3. Equivalent mode activity $a_{e q}$ for the different parts of the drive train

\begin{tabular}{lllll}
\hline Part & pump & diesel & propeller & generator \\
\hline$a_{e q}$ & 0.0016 & 0.0011 & 0.0017 & 0.0029 \\
\hline
\end{tabular}

Table 4. Modal matrix

\begin{tabular}{lllll}
\hline location & $\omega_{1}$ & $\omega_{2}$ & $\omega_{3}$ & $\omega_{4}$ \\
\hline pump & 0.0089 & -0.0104 & 0.0056 & -0.0025 \\
diesel & 0.0089 & 0 & -0.0092 & 0.0115 \\
propeller & 0.0089 & 0.0069 & -0.0067 & -0.0122 \\
generator & 0.0089 & 0.0148 & 0.0162 & 0.0061 \\
\hline
\end{tabular}

Table 5. Absorber parameters

\begin{tabular}{llll}
\hline$m_{h}\left(k g m^{2}\right)$ & $k_{h}\left(\frac{N m}{r a d}\right)$ & $c_{h}\left(\frac{N m s}{r a d}\right)$ & $\omega_{h}\left(\frac{r a d}{s}\right)$ \\
\hline 300 & 6346 & 1533 & 14.54 \\
\hline
\end{tabular}

can obtain on the vibration amplitude caused by a step in the speed of the diesel engine, while Figure 10 shows the obtained reduction when engaging the pump. For the system without absorber, one can see clear that the transient vibrations are much lower for a diesel excitation compared to a pump excitation. This is due to the fact that the speed pick-up is located at the diesel engine. Therefore the absorber is more effective in reducing the vibrations caused by engaging the pump.

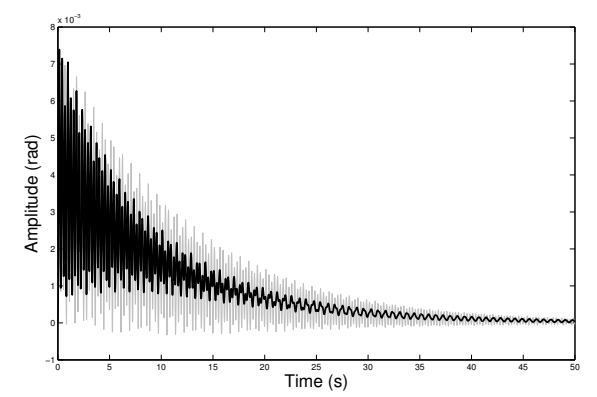

Fig. 9. Reduction of vibrations caused by step of engine's speed with absorber.

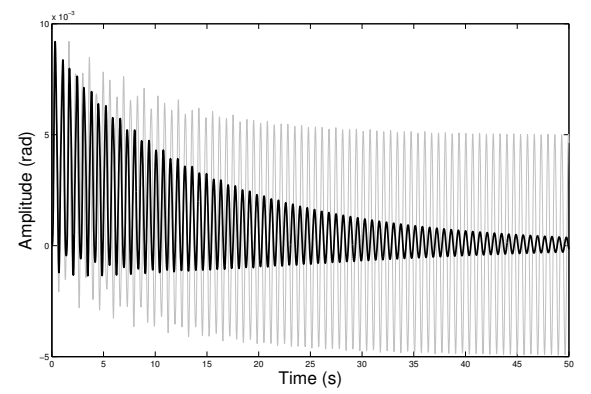

Fig. 10. Reduction of vibrations caused by engaging pump with absorber.

\section{REFERENCES}

Adams, L.M. (2001). Rotating Machinery Vibration, From Analysis to Trouble Shooting. Marcel Dekker Inc.. New York.

Den Hartog, J.P. (1956). Mechanical Vibrations. McGraw-Hill. New York.

Frahm, H. (1911). Device for damping vibrations of bodies. U.S. patent 989,958.

Guyan, R.J. (1965). Reduction of stiffness and mass matrices. AIAA Journal 3(2), 380.

Harris, C.M. and A.G. Piersol (2001). Harris' Shock and Vibration Handbook. Mcgraw-hill. New York.

Koronev, B.G. and L.M. Reznikov (1993). Dynamic Vibration Absorbers, Theory and Technical Applications. Wiley. New York.

Ozer, M.B. and T.J. Royston (2005). Extending den hartog's vibration absorber technique to multi-degree-of-freedom systems. Journal of Vibration and Accoustics 127, 341-350.

Petit, F. and M. Loccufier (2006). Interaction of the governor behaviour and the torsional vibrations of a ship propulsion systems. in Proceedings of the International Conference on Ship and Shipping research pp. 7401-7412.

Rade, D.A. and S. Valder (2000). Optimisation of dynamic vibration absorbers over a frequency band. Mechanical Systems and Signal Processing 14(5), 679-690.

Vance, J.M. (1988). Rotordynamics of Turbomachinery. Wiley. New York.

Vethecan, J.K. and A. Subic (2002). Measures of location effectiveness of vibration absorbers. International Journal of Acoustics and Vibration 7(3), 131-140.

Walsh, P.L. and J.S. Lamancusa (1992). A variable stiffness vibration absorber for minimization of transient vibrations. Journal of Sound and Vibration 158(2), 195-211.

Wilson, W.K. (1942). Practical Solution of Torsional Vibration Problems. John Wiley and Sons Inc.. New York. 